\title{
Photon Is Shortened Name for Electromagnetic Field but Not a Particle
}

\section{Marat Vladimirovich Guryev}

Moscow, Russia

Email:mvguryev@mail.ru

How to cite this paper: Guryev, M.V. (2018) Photon Is Shortened Name for Electromagnetic Field but Not a Particle. Journal of Applied Mathematics and Physics, 6, 1883-1885.

https://doi.org/10.4236/jamp.2018.69159

Received: August 24, 2018

Accepted: September 17, 2018

Published: September 20, 2018

Copyright (C) 2018 by author and Scientific Research Publishing Inc. This work is licensed under the Creative Commons Attribution International License (CC BY 4.0).

http://creativecommons.org/licenses/by/4.0/ (c) (i) Open Access

\begin{abstract}
We well know that photon concept is self-contradictory because we assume that it is a particle with wave properties. This contradiction insensibly spoils our subconscious thinking. It is shown in the article that photon has no 4-coordinates for work within atomic quantum electrodynamics (QED). This implies that actually photon is not a particle. I draw attention that QED is the most precise theory developed by humankind. It is noticed that terms photon and electromagnetic field in practice are in use as synonyms. These results validate the title of the article and exempt us from contradictions within quantum mechanics.
\end{abstract}

\section{Keywords}

Photon, Quantum Jumps, Quantization, Quant, 4-Coordinates of a Particle, Electromagnetic Field, Quantum Electrodynamics

\section{Introduction}

We will work on the principle that strict mathematical description of results of exact experiments is the main goal of theoretical physics. We will show by the example of photon that verbal explanations based on classical feelings lead us into a blind alley.

It is generally accepted that there exist photons, which are usually considered as particles with wave properties. This implies that we must acknowledge that concept of photon is self-contradictory. It is obvious from the well-known examples in the simple case of double-slit paradox, which is the standard example of these contradictions. We will not consider all large number of articles, which are usually devoted to fruitless analyzes of contradictions in photon concept.

We know long ago about existence of these contradictions and have got accustomed to them. Unfortunately, habitual contradictions in theory are actually 
very harmful because they insensibly spoil our subconscious thinking about micro world (MW). That is, such contradictions spoil the foundations of our thinking in physics.

The goal of this article is to prove its title. The rest of the article is organized as follows. In Section 2 we will show that quantum jumps, concept of quants and quantization do not prove existence of photon as a particle. It is shown in Section 3 that photon has no 4 -coordinates and therefore it is not a particle. Section 4 is a short conclusion.

\section{Quantum Jumps, Quants and Quantization Are Not Arguments for Existence of Photon}

The main argument of proof of existence of photons is the obvious existence of quantum jumps (QJ) at transitions between discrete states of quantum objects. QJ are usually interpreted as arrival or going away of photon from object.

Otherwise, it turns out that QJ does not take place at transitions between continuum states although radiation of light takes part at such transitions also. The simplest example of such transitions is bremsstrahlung, see, e.g. [1]. This implies that $\mathrm{QJ}$ represents exclusively transitions between discrete states of quantum objects (DSQO).

Therefore, we must explain QJ without photons as simple natural event because fluent transitions between discrete states are simply impossible within quantum mechanics. Jump is the only opportunity for such transitions to take place. Therefore, just jumps take place as obligatory result of transitions between DSQO. This implies that existence of $\mathrm{QJ}$ is not strict requirement for existence of just photon concept. We can do without it.

Concept of quant is often considered as some argument for existence of photons. This concept is quite empty because it actually describes merely especial case of electromagnetic field, which appears as result of transition between discrete states.

It is worth noting in this connection that well-known operation of quantization is often considered as additional argument for existence of photons. This operation actually is arbitrary activity and so it has no any validation besides our wish to do something similar to reality. It is merely show of explanation of QJ or concept of quant. Therefore, we must acknowledge that quantization is merely imitation of QJ or photons, as you want.

\section{Photon Has No 4-Coordinates}

I draw the reader's attention that regardless great number of words about photons, see e.g. [2], we cannot meet in serious physical literature at least one photon with its 4-coordinates as it should be for particle, which actually exists. Nobody has noticed until now that photon actually has no its 4-coordinates in all applications for serious calculations. This implies that we should acknowledge that photons actually are not classical particles. 
I hope that the readers do not confuse coordinates of particle, which we speak about above, with 4-coordinates of point in phase space, which define 4-dimential value of electromagnetic field. Just these values are in use in serious calculations.

If we want to avoid confusion with coordinates, we should recall that there exists very good reference theory. This is quantum electrodynamics of atoms and molecules (QED), which is the most precise theory developed by humankind, see, e.g. [3]. In other words, I insist that we do not see 4-coordinates of photon somewhere in calculations within QED.

I hope that the reader does not prepare meanwhile statement that there is a new property of photon as a particle: nonexistence of coordinates. For any case, I remind the reader that there exists Occam's razor.

\section{Conclusions}

No kidding, we have proved that photon does not exist as a particle. In real calculations within QED we write only expressions for electromagnetic field. But, we must recall that we very often at the beginning of calculation write something about "photon". Now we can do the last step and understand real role of word "photon" in physics.

We must acknowledge that in real practice within QED our terms-photon and electromagnetic field are synonyms. This simple fact explains title of the article and exempts us from unneeded discussions.

We will not criticize physicists, who work seriously with photons as particles.

Of course, it would be well if we forgot that photon somewhere was a "particle".

\section{Conflicts of Interest}

The author declares no conflicts of interest regarding the publication of this paper.

\section{References}

[1] Jackson, J.D. (1962) Classical Electrodynamics, Ch. 14. John Wiley and Sons, New York-London.

[2] Agarwal, G.S. (2013) Quantum Optics. Cambridge University Press, Cambridge.

[3] Grant, I.P. (2007) Relativistic Quantum Theory of Atoms and Molecules. Springer, Science-Business Media, New York. https://doi.org/10.1007/978-0-387-35069-1 